\title{
Terminologia, Símbolos e Convenções *
}

Maria Estela Jardim ${ }^{a}$, Mariana P. Pereira ${ }^{b}$

\section{QUANTIDADE DE SUBSTÂNCIA E MOLE}

A grandeza fundamental independente quantidade de substância tem uma importância especial para os químicos, mas até muito recentemente não tinha um nome aceite na generalidade, apesar de ser utilizada a unidade mole. A definição de quantidade de substância, tal como a definição de outras grandezas físicas não tem nada a ver com qualquer escolha de unidades, em particular não tem nada a ver com a mole.

Mole - a mole é a quantidade de substância de um sistema que contenha tantas entidades elementares quantos os átomos de carbono contidos em 0,12 quilogramas de carbono-12. As entidades elementares devem ser especificadas e podem ser átomos, moléculas, ið̃es, electrões, outras partículas ou grupos especificados de partículas.

É incorrecto chamar a $n$ "número de moles", tal como é incorrecto chamar a $m$ "número de quilogramas" ou a $\ell$ "número de metros", pois $n, m$ e $\ell$ são símbolos de grandezas e não são números. É correcto afirmar " $n(\mathrm{Cl})=0,1 \mathrm{~mol}$, em que $n(\mathrm{C} \ell)$ significa a quantidade de C $\ell$ '; contudo, é incorrecto dizer " $\mathrm{n}(\mathrm{C} \ell)$, o número de moles de cloro".

A quantidade de substâncias não é o mesmo que a massa. A quantidade de uma substância e a sua massa, ao contrário do volume, por exemplo, têm em comum a propriedade de serem independentes da temperatura e da pressão. A quantidade de uma dada substância é proporcional à sua massa.

A quantidade de substância é proporcional ao número de entidades elementares dessa substância. $\mathrm{O}$ factor de proporcionalidade é o mesmo para todas as substâncias e o seu inverso é a constante de Avogadro (símbolo $\left.L, N_{\mathrm{A}}\right)^{(1)}$ igual a $(6,022045 \pm 0,000031) \times$ $\times 10^{23} \mathrm{~mol}^{-1}$. A designação número de Avogadro não deve ser utilizada; a constante de Avogadro é uma grandeza fisica com dimensões de

$$
\text { (quantidade de substância) }{ }^{-1} \text {, }
$$

não é um número puro e representa o número de entidades elementares por unidade de quantidade de substância. As unidades elementares devem ser especificadas e podem ser átomos, moléculas, iões, radicais, electrões, grupos de partículas que tenham ou não existência real autónoma, ou misturas.

\section{Exemplos:}

$1 \mathrm{~mol}$ de átomos de cloro tem a massa de $35,453 \mathrm{~g}$ e contém $6,002 \times 10^{23}$ átomos

$1 \mathrm{~mol}$ de moléculas de cloro tem a massa de 70,906 g

$1 \mathrm{~mol}$ de $\mathrm{HgCl}$ tem a massa de $236,04 \mathrm{~g}$

$1 \mathrm{~mol} \mathrm{de} \mathrm{Hg}_{2} \mathrm{Cl}_{2}$ tem a massa de $472,08 \mathrm{~g}$

$1 \mathrm{~mol} \mathrm{de} \mathrm{Hg}^{+}$tem a massa de $200,59 \mathrm{~g}$
$1 \mathrm{~mol}$ de $\mathrm{Hg}_{2}^{2+}$ tem a massa de $401,18 \mathrm{~g}$

$1 \mathrm{~mol} \mathrm{de} 1 / 2 \mathrm{Hg}_{2}^{2+}$ tem a massa de $200,59 \mathrm{~g}$

$1 \mathrm{~mol}$ de $\mathrm{CH}_{3} \cdot$ tem a massa de $15,03 \mathrm{~g}$

$1 \mathrm{~mol}$ de $\mathrm{Cu}_{0,5} \mathrm{Zn}_{0,5}$ tem a massa de $64,46 \mathrm{~g}$

1 mol de electrões tem a massa de $548,60 \mu \mathrm{g}$, contém $6,022 \times 10^{23}$ electrões e tem carga de $-96,49 \mathrm{kC}$

$1 \mathrm{~mol}$ de fotões cuja frequência é $1 \times 10^{14} \mathrm{~Hz}$ tem a energia de $39,90 \mathrm{~kJ}$

$1 \mathrm{~mol}$ de $\mathrm{NaCl}(\mathrm{s})$ tem a massa de $58,443 \mathrm{~g}$

$1 \mathrm{~mol}$ de ar, mistura que contém $78,09 \mathrm{~mol}$ por cento de $\mathrm{N}_{2}$,

$20,95 \mathrm{~mol}$ por cento de $\mathrm{O}_{2}, 0,93 \mathrm{~mol}$ por cento

de $\mathrm{Ar}$, e $0,03 \mathrm{~mol}$ por cento de $\mathrm{CO}_{2}$, tem a massa de $28,964 \mathrm{~g}$

Termos como átomo-grama, molécula-grama, equivalente, equivalente-grama, ião-grama e fórmula-grama caíram em desuso.

\section{Exemplos:}

não deve dizer-se

1 átomo-grama de $\mathrm{He}$

1 molécula-grama de $\mathrm{H}_{2} \mathrm{SO}_{4}$

1 equivalente de $\mathrm{H}_{2} \mathrm{SO}_{4}$

1 ião-grama de $\mathrm{SO}_{2}^{4-}$

1 fórmula-grama de $\mathrm{ZnS}$

1 faraday

1 einstein deve dizer-se

1 mole de $\mathrm{He}$
1 mole de $\mathrm{H}_{2} \mathrm{SO}_{4}$
1 mole de $1 / 2 \mathrm{H}_{2} \mathrm{SO}_{4}$
1 mole de $\mathrm{SO}_{4}^{2-}$
1 mole de $\mathrm{ZnS}$
1 mole de e
12 mole de $\gamma$

\section{BIBLIOGRAFIA}

ASE. (1979). Chemical nomenclature, symbols and terminology. Hatfield, Herts: The Association for Science Education.

IUPAC. (1982). Chemistry International - Special Supplement. Oxford: Pergamon Press.

IUPAC. (1979). Manual of symbols and terminology for physico chemical quantities and units. Oxford: Pergamon Press.

Jardim, M. E. \& Pereira, M. P. Terminologia, simbolos e unidades para grandezas físico-químicas do sistema internacional de unidades, Edição da Sociedade Portuguesa de Quimica e da Livraria Escolar Editora, Lisboa (1985).

McGlashan, M. L. (1971). Physicochemical quantities and units. Monographs for teachers, $n .^{\circ} 15$, London: Royal Institute of Chemistry.

* Neste texto apresentam-se indicaçōes actualizadas sobre terminologia, símbolos e convenções. Na sequência de artigos publicados nos números 12 (pp. 16-17) e 15 (pp. 36-37) inclue-se neste número uma contribuição para o esclarecimento da definição da grandeza físico-química fundamental quantidade de substância e exemplos do emprego da unidade respectiva, designada por mole.

a Dep. Quimica, Faculdade de Ciências de Lisboa.

b Dep. Educação, Faculdade de Ciências de Lisboa.

(1) L. de Loschmidt, que em 1865 determinou o valor desta constante por estudos de teoria cinética de gases. 